\title{
Zur Theorie der Desinfektion.
}

Vorläufige Mitteilung.

Von

R. O. Herzog und R. Betzel.

Mit zwei Kurvenzeichnungen im Text.

(Aus dem Chemischen Institut der Technischen Hochschule in Karlsruhe.)

(Der Redaktion zugegangen am 15. Juni 1910.)

Vor etwa zwei Jahren haben wir Versuche in Angriff genommen, welche eine Aufklärung der physikalischen und chemischen Verhältnisse bei den Desinfektionsvorgängen bezweckten. Davon ausgehend, daß die Antiseptika und die in der Lösung suspendierten Mikroorganismen nicht, wie es bisher geschah, als homogenes, sondern als heterogenes System aufzufassen sind, war der Plan, die Verteilung des Antiseptikums zwischen Lösungsmittel und Organismen zu prüfen. ${ }^{1}$ )

Seither ist dasselbeProblem von ähnlichen Gesichtspunkten aus von verschiedener Seite in Angriff genommen und bearbeitet worden. Zuerst hat $\mathrm{H}$. Bechhold ${ }^{2}$ ) die Frage behandelt, sich teils auf Adsorptionsversuche im allgemeinen, teils auf Versuche über Desinfektion stützend, insbesondere boten die Arbeiten von Krönig und $\mathrm{Paul}^{8}$ ) geeignetes Material, welches

1) Die Versuche mußten wegen Militärdienstes Herrn Betzels für ein Jahr unterbrochen werden. Über unsere vorläufigen Ergebnisse, soweit reichend, wie sie hier mitgeteilt werden, wurde am 18. Juni 1909 in der Karlsruher Chemischen Gesellschaft berichtet. Vgl. Chem. Ztg., 1909, Nr. 78.

2) Intern. Kongreß f. angew. Chem., Mai-Juni 1909. - Zeitschrift f. Chem. u. Ind. d. Koll., Bd. V, S. 22 (1909).

${ }^{3}$ ) Zeitschrift f. Hyg., Bd. XXV, S. 1 (1897). - Ferner Paul, Entwurf z. einheitl. Wertbest. Chem. Desinfektionsmittel von Th. Paul, Berlin 1901. 
auch H. Morawitz ${ }^{1}$ ) und H. Freundlich ${ }^{2}$ ) ihren Betrachtungen zugrunde legten. Soeben wandten endlich Th. Paul, G. Birstein und A. ReuB ${ }^{3}$ ) denselben Gedanken gelegentlich neuer Versuche mit Sauerstoff als Desinfektionsmittel an.

Die genannten Verfasser gingen alle davon aus, in irgend einer Weise auf Grund theoretischer Überlegungen oder rechnerisch die Mitwirkung von Adsorptionsvorgängen festzustellen, eine Zwangslage, solange man mit Bakterien arbeitet. Um diese Schwierigkeit zu vermeiden, haben wir als Mikroorganismen Hefe angewandt, die nach hinreichender Einwirkung des gelösten Antiseptikums durch Zentrifugieren von der Lösung getrennt wurde.

Die vorläufigen, im folgenden kurz mitgeteilten Versuche bezweckten nur die Feststellung, wieviel bei variierter Giftkonzentration von derselben Hefemenge aufgenommen wird. Weder auf die Frage, in welcher Beziehung die aufgenommene Giftquantität zu der eigentlich wirksamen steht, noch darauf, inwieweit es sich um reversible Vorgänge handelt oder nicht, oder auf den Einfluß der Temperatur usw. wird vorerst eingegangen, doch sollen fortgesetzte Versuche gerade auch nach dieser Richtung Aufklärung bringen.

Die Antiseptika, welche in relativ verdünnter Lösung auf Mikroorganismen wirken, reagieren, soweit bekannt, entweder mit den Proteinen oder sie besitzen ein erhebliches Löslichkeitsvermögen für Fette und sogenannte Lipoidstoffe oder umgekehrt diese für sie. Von den Giftstoffen letzter Art wurde vorwiegend Chloroform untersucht, von Stoffen, die rorwiegend gegenüber Eiweiß reaktionsfähig sind - sei es chemisch, sei es, daß sie auf deren kolloidale Lösungen einwirken Salze, von denen hier nur über Silbernitrat berichtet werden soll, ferner Formaldehyd. Auch Phenol wurde geprüft.

Die hauptsächliche und zum Teil sehr zeitraubendeSchwierigkeit der Arbeit lag in der Erzielung einwandfreier Analysen;

1) Kolloidchem. Beihefte, Bd. I, S. 301 (1910).

2) Das., S. 317.

s) Biochem. Zeitschrift, Bd. XXV, S. 367 (1910). 
über die schließlich angewandte Methodik wird in einer späteren Mitteilung berichtet werden.

$5 \mathrm{~g}$ Preßhefe werden mit Chloroformwasser von dem angegebenen Gehalt zusammen gebracht (Volumen $99 \mathrm{ccm}$ ) und 4 Stunden geschüttelt.

Angewandte Menge

Chloroform in $\mathbf{g}$

0,5347

0,4221

0,2814

0,2814

0,1407

0,0563
Von der Hefe aufgenommene

Chloroformmenge in $\mathbf{g}$

0,0876

$0,057 \tilde{5}$

0,0372

0,0355

0,0122

0,0047

In einem anderen Versuche (mit anderer Hefe usw.) werden erhalten (eine dritte zwischen beiden angegebenen Konzentrationen liegende Bestimmung fällt soweit heraus, daß ein grober Versuchsfehler wahrscheinlich ist):

$\begin{array}{cc}\text { Angewandte Menge } & \text { Von der Hefe aufgenommene } \\ \text { Chloroform in } \mathrm{g} & \text { Chloroformmenge in } \mathrm{g} \\ 0,4221 & 0,0541 \\ 0,1688 & 0,0167\end{array}$

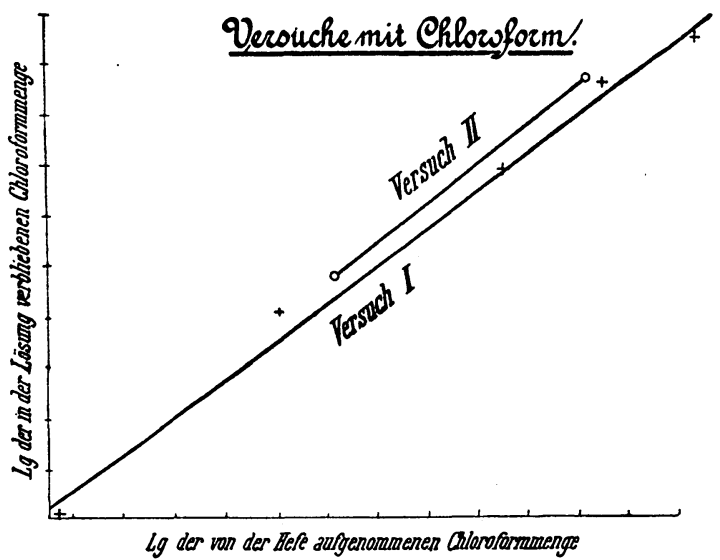

Der für die Adsorption berechnete Exponent beträgt 1,4.

Die folgenden voneinander vollständig unabhängigen Versuchsreihen sind mit Silbernitrat angestellt, bei der ersten beträgt die Menge der zugefügten Hefe $5 \mathrm{~g}$, das Volumen der 
Lösung $100 \mathrm{ccm}$; bei der zweiten die Hefe $10 \mathrm{~g}$, das Volumen $107,5 \mathrm{ccm}$.

Auffallenderweise sind die Gleichgewichtskurven nicht parallel; der Exponent der Adsorptionskurve ist im ersten Fall ungefähr $1 / 2$, im zweiten $1 / 3$, beide liegen aber innerhalb der von Biltz und Freundlich für die Adsorption als Regel angegebenen Grenzen.

1. Versuch.

$\begin{array}{cc}\text { Ursprüngliche Menge } & \begin{array}{c}\text { Von der Hefe aufgenommene } \\ \text { Silbernitratmenge in } \mathrm{g}\end{array} \\ \text { Silbernitrat in } \mathrm{g} & 0,643 \\ 8,481 & 0,426 \\ 4,241 & 0,326 \\ 2,827 & 0,276 \\ 2,120 & 0,240 \\ 1,696 & 0,175 \\ 0,848 & \end{array}$

2. Versuch.

Ursprüngliche Menge

Silbernitrat in $\mathbf{g}$

$$
\begin{aligned}
& 8,168 \\
& 4,084 \\
& 2,723 \\
& 2,042 \\
& 1,6336 \\
& 0,8168
\end{aligned}
$$

Von der Hefe aufgenommene

Silbernitratmenge in $\mathbf{g}$

$$
\begin{aligned}
& 0,654 \\
& 0,533 \\
& 0,454 \\
& 0,409 \\
& 0,364 \\
& 0,293
\end{aligned}
$$

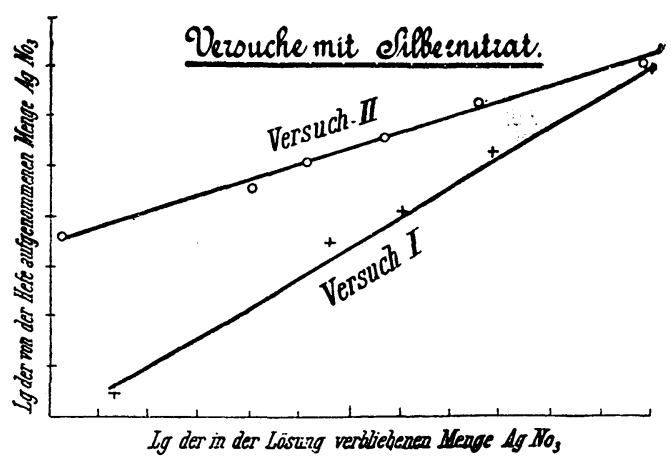

Ein ganz anderes Bild zeigen die Versuche mit Formaldehyd. 
1. Versuch.

Formaldehyd angewandt

in $\mathrm{g}$

0,835

0,527

0,263

0,263

$\left.0,132^{1}\right)$
Von der Hefe aufgenommene Menge

in $\mathrm{g}$

0,025

0,032

0,030

0,029

0,029

2. Versuch.

Formaldehyd angewandt in $\mathrm{g}$

2,514

1,675

0,838

0,838

$0,447^{1)}$
Von der Hefe aufgenommene Menge

in $\mathrm{g}$

0,047

0,036

0,044

0,036

0,035

Die angeführten Versuche zeigen also, daß innerhalb der Fehlergrenzen der Methode eine gewisse Hefemenge immer eine bestimmte, von der Konzentration unabhängige Formaldehydmenge bindet.

Versuche, die mit Phenol ${ }^{2}$ ) angestellt wurden, ließen innerhalb 1,87- bis 0,187\% \% igen Lösungen keine Aufnahme des Antiseptikums durch die Hefe erkennen.

Die angeführten Versuche zeigen also, daß Silbernitrat von Hefe so aufgenommen wird, wie dies bei einem Adsorptionsprozeß der Fall sein müßte; Chloroform zeigt dasselbe Verhalten, nur fällt der Adsorptionsexponent aus den gewöhnlichen Fragen heraus - was freilich auch sonst schon bei typischen Adsorptionsvorgängen beobachtet wurde -; von Formaldehyd wird stets eine konstante Menge gebunden, unabhängig von der Konzentration; bei Phenol konnte Aufnahme durch die Organismen nicht nachgewiesen werden. Die Versuche werden in der oben angedeuteten Richtung fortgesetzt.

1) Bei einer noch geringerer Konzentration ist eine offenbar durch einen Versuchsfehler entstellte abweichende Zahl gefunden worden.

2) Vgl. über die Phenolwirkung Reichel, Biochem. Zeitschrift, Bd. XXII, S. 149, 177, 201 (1909). 